\title{
Finding Fair Negotiation Algorithms to Reduce Peak Electricity Consumption in Micro Grids
}

\author{
Simon T. Powers ${ }^{1}$, Oscar Meanwell ${ }^{1}$, and Zuansi Cai ${ }^{2}$ \\ ${ }^{1}$ School of Computing, Edinburgh Napier University, Edinburgh EH10 5DT, U.K. \\ ${ }^{2}$ School of Engineering and the Built Environment, Edinburgh Napier University, Edinburgh \\ EH10 5DT, U.K. \\ S.Powers@napier.ac.uk
}

\begin{abstract}
Reducing peak electricity consumption is important to maximise use of renewable energy sources, and reduce the total amount of capacity required on a grid. Most approaches use a centralised optimisation algorithm run by a utility company. Here we develop a decentralised approach, where agents represent the interests of a household, and negotiate over when to run various appliances. We have developed an experimental framework that allows users' perceived fairness of different negotiation algorithms to be evaluated.
\end{abstract}

Keywords: Smart Grid; Resource Allocation; Load Balancing

\section{$1 \quad$ Introduction}

With the increasing affordability of renewable energy sources such as solar panels and wind turbines, more and more households and communities are starting to become prosumers that produce their own electricity as well as consuming it from the national grid. Groups of households and organizations that jointly invest in renewable sources are known as community energy systems [1]. A community energy system establishes its own micro grid - the electricity that it produces is distributed amongst its members, reducing the amount that they have to buy in from external energy suppliers. Financially, it is advantageous for members to use as much of the energy that the system produces as they can, because the price they get for selling it to the national grid is much lower than the cost of buying in energy to meet their demand.

A key problem that community energy systems then face is load balancing. If every household uses their appliances (e.g. electric heaters, dishwashers) at the same time, then the energy the community produces will be unable to meet this demand, and they will be forced to buy in a large amount from the national grid, possibly supplied by non-renewable sources. A solution to this problem lies in reducing peak consumption by spreading usage out throughout the day. In principle, this can flatten consumption if every household's usage is spaced out to match production from the renewable source. However, each household also has their own preferences for when they would like to use their appliances. This means that if households are going to be motivated to subject 
their usage to load balancing, then they need to both see a reduction in peak consumption and hence cost to them, and perceive the load balancing process as treating them fairly [2].

To address this problem, we propose the use of a multi-agent system embedded in Home Area Networks. A Home Area Network connects smart appliances and control switches to a smart meter that can turn them on and off [3]. In our design, each household is represented by an agent. A household enters their preferences for the timeslots in which they would like to run various appliances. Their agent then negotiates with the agents representing other households in the system to produce a schedule for running their appliances, which is then sent to their smart meter. We are interested in which kinds of negotiation algorithms households perceive as treating them fairly.

\section{Main purpose}

We assume that for a given day the total amount of energy that all households in the community energy system wish to consume is divided into hourly timeslots of equal Kilowatt hour capacities. In a more realistic setting, the size of each timeslot would be based on the predicted amount of energy that the renewable energy source would produce during that time period.

We are currently investigating users' perceptions of the allocations resulting from three negotiation algorithms. The first allocates timeslots randomly to users. The second involves running an English auction to allow households to bid for timeslots. Each household is allocated a number of virtual credits that they can use to bid for timeslots for the following day. In the initial version of the system all households are allocated the same amount of credits that they can use for bidding. However, it is possible to allocate different amounts of credits to households, for example based on their size, or the amount of money they have invested into the community energy system. These would represent fairness in the distribution of resources based on need or effort, respectively [2]. The bidding process could be done interactively by users themselves, or on their behalf by their agent, depending on the level of involvement that the household wishes to have.

The third algorithm, inspired by work on social capital in multi-agent systems [4], involves an initially random allocation of timeslots to households. Agents can then propose timeslot exchanges to other agents. In the base version, agents only accept a proposed exchange if that exchange will allow one of their own timeslot preferences to be satisfied. In a second version, agents gain reputation points by accepting an exchange that is not beneficial to them. These reputation points can be cashed in on the following days, by allowing the agent to exchange a certain number of reputation points for the guaranteed allocation of a particular timeslot. As with the auction mechanism, this algorithm could be fully automated by the agents, or could allow households to propose exchanges interactively. 


\section{Demonstration}

We have developed a prototype of an application that allows users to enter their preferences for the timeslots in which they would like to run various appliances (Figure 1), and take part in one of the three algorithms (Figure 2). We then solicit feedback from the user about how satisfied they were with the allocations that they received from that algorithm (Figure 3). The experimenter can choose the number of timeslots that users can allocate their appliances to on a day, the types of appliances that can be allocated, the negotiation algorithm that will be used, and the number of days that will be simulated. A number of users can then be invited into the laboratory. We record users' preferences, the resulting allocations, and users' satisfaction with the allocation. At the end of the experiment each user is shown the allocations that every other user received on each day along with their preferences, and are asked to rate the fairness of the series of allocations.

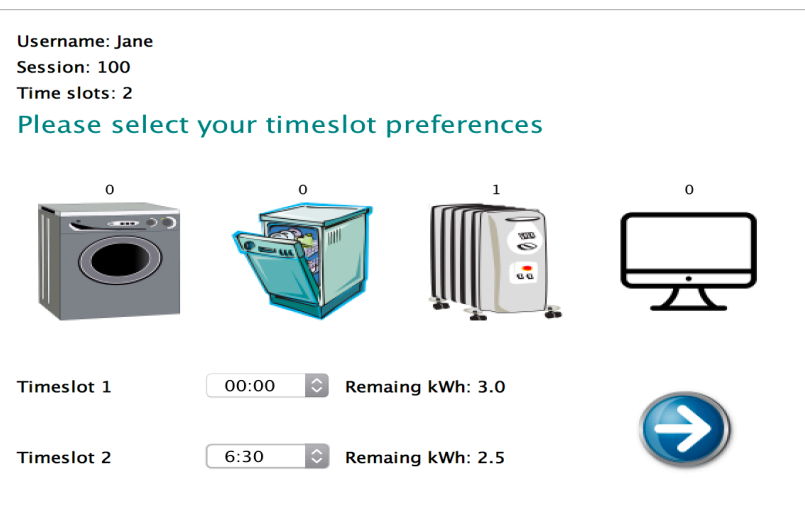

Fig. 1. Here the user has chosen 00:00-01:00 and 06:30-07:30 as slots in which they would like to run appliances that can be time delayed. Each slot has up to 4 kilowatt hours of electricity available for the user to distribute between various appliances as they choose. In this example, the user wishes to run their electric heater for 1 hour at 06:30.

Please bid for your preferred timeslots

Please bid for the timeslot

OO: $\mathrm{OO}$

Credits Available: 4.0

Current Bids: 4

Current highest bid: 0.5

Enter your max bid

Submit Bid

Fig. 2. The user then bids for their chosen timeslots if using the auction algorithm. 


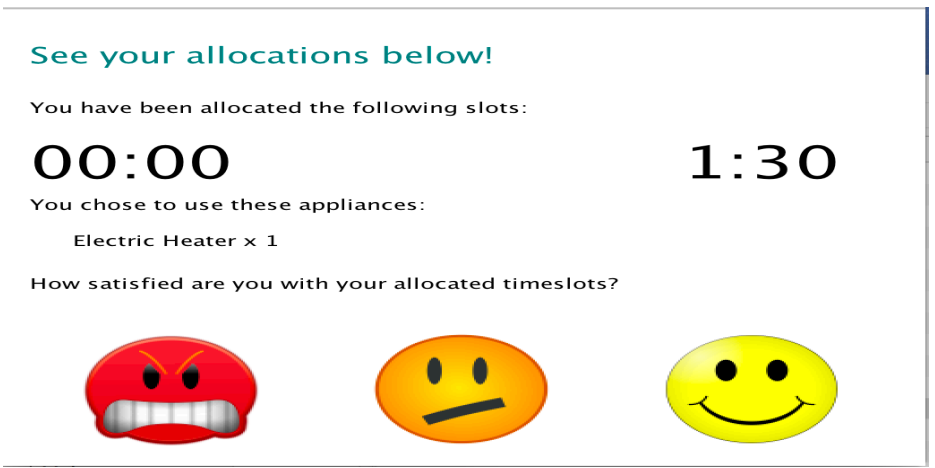

Fig. 3. Finally, the user is shown the resulting allocations from the negotiation algorithm and asked to rate their satisfaction.

\section{Conclusions}

Load balancing to reduce peak consumption is important to allow maximal use of renewable energy sources. It is also important for energy companies supplying through the national grid, who have to provide excess capacity to meet peak demand. In contrast to centralised optimisation processes run by a utility company, we have proposed a system based on decentralised optimisation, where each household is represented by an agent responsible for meeting its preferences, and that optimises by negotiating with other agents. In addition to monetary incentives, we focus on the perceived fairness of the load balancing algorithm. This is important given that behavioural economics experiments demonstrate that people have a preference for fairness in addition to monetary incentives [5].

\section{References}

1. van der Schoor, T., Scholtens, B.: Power to the people: Local community initiatives and the transition to sustainable energy. Renew. Sustain. Energy Rev. 43, 666-675 (2015).

2. Pitt, J., Schaumeier, J., Busquets, D., Macbeth, S.: Self-Organising Common-Pool Resource Allocation and Canons of Distributive Justice. In: 2012 IEEE Sixth International Conference on Self-Adaptive and Self-Organizing Systems. pp. 119-128 (2012).

3. Shao, S., Pipattanasomporn, M., Rahman, S.: An approach for demand response to alleviate power system stress conditions. In: 2011 IEEE Power and Energy Society General Meeting. pp. 1-7 (2011).

4. Petruzzi, P.E., Busquets, D., Pitt, J.: Self Organising Flexible Demand for Smart Grid. In: 2013 IEEE 7th International Conference on Self-Adaptation and Self-Organizing Systems Workshops. pp. 21-22 (2013).

5. Fehr, E., Schmidt, K.M.: A Theory of Fairness, Competition, and Cooperation. Q. J. Econ. 114, 817-868 (1999). 